\title{
Different definitions of CpG island methylator phenotype and outcomes of colorectal cancer: a systematic review
}

\author{
Min Jia ${ }^{1}$, Xu Gao ${ }^{1}$, Yan Zhang ${ }^{1}$, Michael Hoffmeister ${ }^{1}$ and Hermann Brenner ${ }^{1,2^{*}}$
}

\begin{abstract}
Contradictory results were reported for the prognostic role of $\mathrm{CpG}$ island methylator phenotype (CIMP) among colorectal cancer (CRC) patients. Differences in the definitions of CIMP were the most common explanation for these discrepancies. The aim of this systematic review was to give an overview of the published studies on CRC prognosis according to the different definitions of CIMP. A systematic literature search was performed in MEDLINE and ISI Web of Science for articles published until 3 April 2015. Data extraction included information about the study population, the definition of CIMP, and investigated outcomes. Thirty-six studies were included in this systematic review. Among them, 30 studies reported the association of CIMP and CRC prognosis and 11 studies reported the association of CIMP with survival after CRC therapy. Overall, 16 different definitions of CIMP were identified. The majority of studies reported a poorer prognosis for patients with CIMP-positive (CIMP+)/CIMP-high (CIMP-H) CRC than with CIMP-negative (CIMP-)/CIMP-low (CIMP-L) CRC. Inconsistent results or varying effect strengths could not be explained by different CIMP definitions used. No consistent variation in response to specific therapies according to CIMP status was found. Comparative analyses of different CIMP panels in the same large study populations are needed to further clarify the role of CIMP definitions and to find out how methylation information can best be used to predict CRC prognosis and response to specific CRC therapies.
\end{abstract}

Keywords: Colorectal cancer, CpG island methylator phenotype, Prognosis, Chemotherapy

\section{Background}

With estimated numbers of approximately 1.4 million new cases per year, colorectal cancer (CRC) is the third most commonly diagnosed cancer in males and the second most common in females globally [1]. The 5-year survival rate is less than $65 \%$ [2].

CpG island methylator phenotype (CIMP) which was originally introduced by Toyata and colleagues in 1999 [3] is characterized by simultaneous hypermethylation of numerous $\mathrm{CpG}$ islands surrounding the promoter regions of several genes [4]. Methylation of $\mathrm{CpG}$ islands in the promoter of tumor-suppressor genes could physically inhibit binding of transcription factors [5]. By transcriptional silencing of these genes, CIMP is believed to contribute to the onset and progression of CRC $[6,7]$.

\footnotetext{
*Correspondence: h.brenner@dkfz-heidelberg.de

${ }^{1}$ Division of Clinical Epidemiology and Aging Research, German Cancer

Research Center (DKFZ), Heidelberg, Germany

${ }^{2}$ German Cancer Consortium (DKTK), Heidelberg, Germany
}

However, definitions of CIMP varied widely between studies with respect to methylation loci considered and laboratory methods used to determine methylation.

Previous studies showed that CIMP is associated with altered molecular and clinical characteristics $[8,9]$. However, the prognostic effect of CIMP in CRC was not consistent in previous studies. Recently, Juo and colleagues [10] conducted a meta-analysis supporting the hypothesis that CIMP positivity predicts poorer survival among patients with CRC. However, this meta-analysis did not take the differences in the definitions of CIMP into consideration. The aim of this systematic review was to give an overview of the published studies on CRC outcomes according to the definitions of CIMP and to provide a deeper understanding of the importance of CIMP definition in the evaluation of CRC prognosis. 


\section{Methods}

\section{Search strategy}

Our systematic literature search was performed in accordance with PRISMA recommendations [11]. PubMed and ISI Web of Knowledge databases were used for the search of relevant articles from inception to 3 April 2015, using neither filters nor language restrictions. The combination of keywords used was [colorectal (or) colon (or) rectum] (and) [cancer (or) neoplasm (or) carcinoma (or) adenoma (or) malignancy] (and) [methylation] (and) [prognosis (or) prognostic (or) survival (or) follow up (or) mortality (or) long term] (and) [CIMP (or) CpG island methylator phenotype]. A search for additional relevant studies was carried out in the reference lists of the identified studies.

\section{Eligibility criteria}

After deleting duplicate articles, each title and abstract was checked for relevant content. Only studies published in English language that were conducted in human beings, measuring methylation in a biological sample, were included. Studies not relevant to the topic, such as studies only testing global DNA methylation level in human blood or cell lines, were excluded. Since detailed information was needed for further review, studies with only conference abstract or not reported in original articles were also excluded. As this review focused on CRC outcomes according to different CIMP definitions, studies not reporting the specific methylation markers used for the definition of CIMP or the criteria for the classification of CIMP categories and studies that did not have survival data of CRC patients were excluded after full-text review.

\section{Data extraction}

Data were extracted from the eligible studies by two investigators (MJ and XG) independently into data extraction tables. Any disagreement was resolved by consensus after further review of the original text. The following data items were extracted: study population information (authors, year of publication, country, patient population, number of subjects, age, sex, and follow-up time), information about the definition of CIMP (methylation loci, classification of CIMP, prevalence of CIMP, and laboratory method for methylation analysis), and outcome data ( $p$ value and hazard ratio (HR) and $95 \%$ confidence intervals $(95 \% \mathrm{CI})$ ) including associations with overall survival (OS), disease-specific survival (DSS), disease-free survival (DFS), and recurrence-free survival (RFS). Given the importance of potential confounding factors, we extracted data from the most comprehensively adjusted model only when results from different models were reported. If no adjusted results were available, we included the unadjusted results.

\section{Results}

\section{Literature search result}

The literature search found 394 articles in total (Fig. 1). After removal of 125 duplicates, 269 titles and abstracts were screened for potentially relevant articles. Following exclusion of 193 non-relevant articles and 31 nonoriginal articles, 45 studies were left for full-text review, and two additional studies related to the topic were found by cross-referencing. These 47 studies included studies investigating the association between CIMP and prognosis of CRC $(n=41)$ and studies investigating survival after specific CRC therapies according to CIMP status $(n=11)$. Among the 41 studies on CRC prognosis, 11 studies were excluded after full-text assessment for the following reasons: studies with no comparison of different CIMP categories with respect to prognosis $(n=7)$; studies without information about survival $(n=3)$; and studies with too small sample size to carry out meaningful survival analyses $(n=1)$. Finally, we included 30 studies on the association of CIMP with CRC prognosis and 11 studies on the association of CIMP with survival according to CRC therapy.

\section{Study population characteristics}

An overview of the 30 included studies on CIMP and prognosis of CRC $[4,8,12-40]$ is shown in Additional

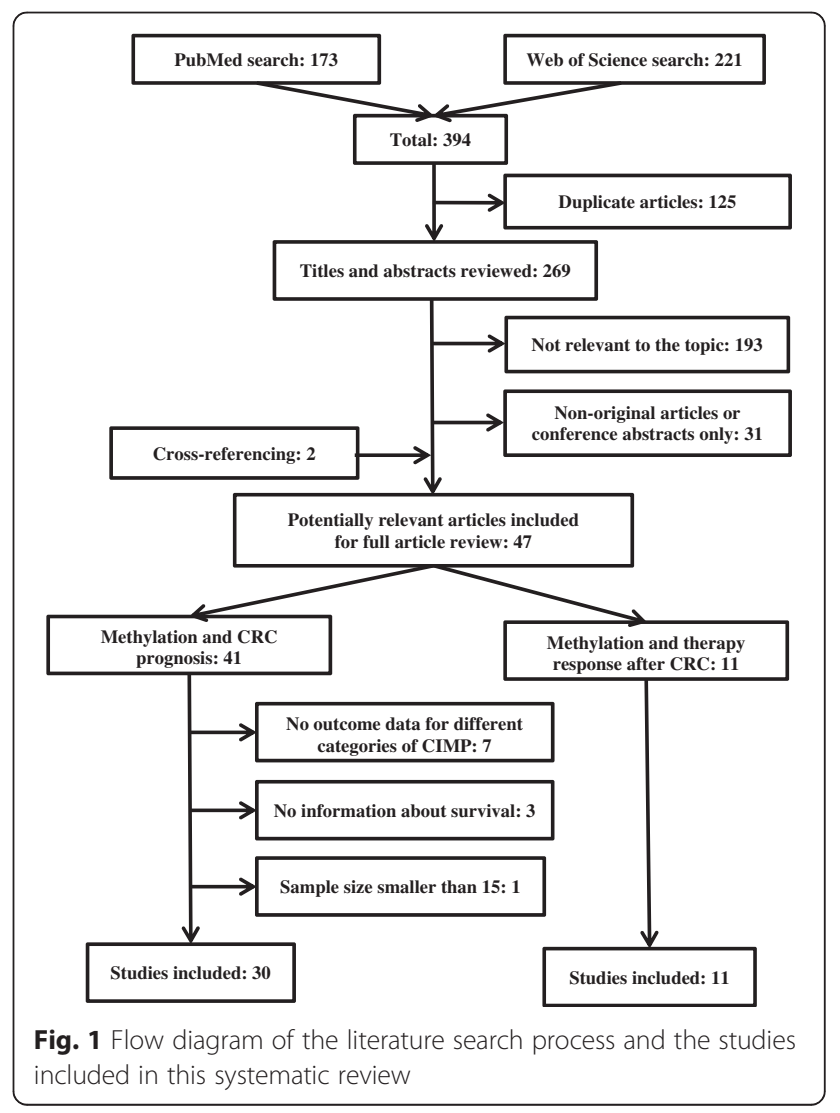


file 1: Table S1. Most studies investigated colon and rectal cancer patients (26/30), whereas others only focused on colon cancer patients $(4 / 30)$ or rectal cancer patients $(1 / 30)$. Some of the studies were restricted to specific stages of CRC $(n=5)$ or specific molecular subtypes of CRC (3/30). OS was assessed in most of the studies, while others assessed DFS, DSS, and RFS as the primary end point. The 11 studies on survival after specific CRC therapies according to CIMP status [4, 25, 34-36, 40-45] were mainly including stage II or stage III CRC patients (Additional file 2: Table S2). Six of these 11 studies focused on patients with surgery alone and patients with surgery and adjuvant chemotherapy [4, 25, 34, 36, 40, 41]. One study [35] investigated two patient groups with different chemotherapy regimens after surgery. The remaining four studies enrolled patients treated with chemotherapy only or with chemotherapy after surgery [42-45].

\section{CIMP definition}

Gene panels used for the definition of CIMP varied between the studies (Tables 1 and 2). CIMP was either classified in two groups (CIMP positive (CIMP+) and CIMP negative (CIMP-)) or in three groups (CIMP-high (CIMP-H), CIMP-low (CIMP-L), and CIMP-negative $(\mathrm{CIMP}-\mathrm{N}))$. In the 16 different marker panels used for the definition of CIMP, the number of methylation loci included ranged between 5 and 15. Additionally, definitions 1 and 2 and definitions 3 and 4 used different cutoff values for CIMP-H or CIMP+. Prevalence of CIMP+ or CIMP-H varied between different CIMP definitions and the patient samples investigated (6.4-48.5\%). The difference of prevalence of CIMP+ or CIMP-H among studies using the same CIMP definition might be due to different patient races, different laboratory methods, and different subgroups of CRC patients. Regarding the laboratory method used to analyze the methylation of genes, methylation-specific PCR (MSP, $n=15$ ) [46] and MethyLight $(n=16)$ [47] were the most frequently used. Other methods such as pyrosequencing $(n=2)$, MassARRAY $(n=1)$ [22, 48], MS-HRM (methylationsensitive high-resolution melting, $n=1$ ) [49], and COBRA (combined bisulfite restriction analysis, $n=1$ ) [43] were used by few studies only (Additional file 3: Table S3 and Additional file 4: Table S4). Where reported, DNA was extracted from formalin-fixed and paraffin-embedded (FFPE) tissue in the majority of studies (Additional file 3: Table S3 and Additional file 4: Table S4).

\section{CIMP definition and CRC prognosis}

As only very few studies investigated the same CIMP definition in association with the same outcome and in similar subgroups, it was not meaningful to perform meta-analyses. According to the available studies, no obvious impact of CIMP definition on the prognostic value of CIMP was found (Tables 3, 4, and 5, Additional file 5: Table S5). Most of the studies reported poorer outcome for CIMP+ patients compared with CIMPpatients, but studies often failed to reach statistical significance due to an insufficient sample size. Some studies just reported univariate results without adjustment for important confounders. Results for CIMP-L were reported for few studies only. Again, the majority of studies showed a trend towards reduced survival which was though not consistent and often not statistically significant. Comparison of the impact of CIMP across studies was hampered by the different definitions of CIMP used. Age and tumor stage were adjusted for in the multivariate analysis of most studies, but BRAF mutation, KRAS mutation, and microsatellite instability (MSI) were assessed as confounding variables only in a few studies. CIMP+ was reported as an independent prognostic for poorer survival in rectal cancer patients compared to CIMP-, both for OS and DFS [20, 28], and was associated with poorer survival also among CRC patients with MSI $[8,23,26]$.

\section{Survival after specific CRC therapies according to CIMP status}

The limited number of studies on survival after specific CRC therapies did not show a clear pattern of effect modification of chemotherapy success by CIMP status (Table 6). Among patients with CIMP+, three studies showed no statistically significant differences on survival for patients who were treated with surgery and chemotherapy compared with surgery alone. Results were inconsistent among patients with CIMP-. Shiovitz et al. [35] reported potentially enhanced survival after surgery $+\mathrm{FU}+\mathrm{LV}+\mathrm{IFL}$ therapy compared to patients receiving surgery + FU + LV therapy only among CIMP+ patients (HR, 0.62; 95 \% CI, 0.37-1.05), while CIMP- patients had a lower DFS with the combination therapy (HR, 1.38; 95 \% CI, 1.00-1.89).

\section{Discussion}

Based on the results of this review, predominantly poorer survival was observed in patients with CIMP+ or CIMP-H. Regarding CIMP definitions, no obvious difference could be observed in the prognostic value of the 16 different CIMP panels due to the high heterogeneity of CIMP definitions, subgroups, and outcomes investigated. A plausible reason might be that CIMP in most studies contains overlapping gene markers to various extents. Most of the studies chose commonly used CIMP-related gene markers to define CIMP except for the study by Hokazono et al. [33]. Among the 15 included in CIMP definitions, MLH1, MINT1, CACNA1G, and p16 were 
Table 1 CIMP definitions and prevalence in studies on colorectal cancer prognosis

\begin{tabular}{|c|c|c|c|c|c|c|c|c|c|c|c|c|c|c|c|c|c|}
\hline \multirow[t]{3}{*}{ Definition } & \multirow{3}{*}{ First author (year) } & \multicolumn{11}{|c|}{ Common CIMP genes } & \multirow[t]{3}{*}{ Other CIMP genes } & \multicolumn{3}{|c|}{ CIMP category } & \multirow{3}{*}{$\begin{array}{l}\mathrm{CIMP}+/-\mathrm{H} \\
\text { prevalence }\end{array}$} \\
\hline & & \multirow[b]{2}{*}{ CACNA1G } & \multirow[b]{2}{*}{ IGF2 } & \multirow[b]{2}{*}{ NEUROG1 } & \multirow[b]{2}{*}{ RUNX3 } & \multirow[b]{2}{*}{ SOCS1 } & \multirow[b]{2}{*}{ CRABP1 } & \multirow[b]{2}{*}{ MLH1 } & \multirow[b]{2}{*}{ p16 } & \multirow[b]{2}{*}{ MINT1 } & \multirow[b]{2}{*}{ MINT2 } & \multirow[b]{2}{*}{ MINT31 } & & \multirow{2}{*}{$\begin{array}{l}\text { CIMP+ } \\
\text { CIMP-H }\end{array}$} & \multicolumn{2}{|l|}{ CIMP- } & \\
\hline & & & & & & & & & & & & & & & $\overline{C I M P-L}$ & CIMP-N & \\
\hline \multirow[t]{4}{*}{$\bar{D} 1$} & Samowitz (2005) [13] & & & & & & & + & + & + & + & + & & $2 / 5$ & $0-1 / 5$ & & $24.6 \%$ \\
\hline & Lee (2008) [16] & & & & & & & + & + & + & + & + & & $2 / 5$ & $0-1 / 5$ & & $31.3 \%$ \\
\hline & Samowitz (2009) [20] & & & & & & & + & + & + & + & + & & $2 / 5$ & $0-1 / 5$ & & $11.9 \%$ \\
\hline & Ju (2011) [38] & & & & & & & + & + & + & + & + & & $2 / 5$ & $0-1 / 5$ & & $24.4 \%$ \\
\hline \multirow[t]{7}{*}{ D 2} & Kalady (2009) [17] & + & + & + & + & + & & & & & & & & $3 / 5$ & $0-2 / 5$ & & $21.8 \%$ \\
\hline & Sanchez (2009) [21] & + & + & + & + & + & & & & & & & & $3 / 5$ & $0-2 / 5$ & & $21.2 \%$ \\
\hline & $\operatorname{Min}(2011)$ [25] & + & + & + & + & + & & & & & & & & $3 / 5$ & $1-2 / 5^{a}$ & $0 / 5$ & $13.9 \%$ \\
\hline & Donada (2013) [40] & + & + & + & + & + & & & & & & & & $3 / 5$ & $1-2 / 5^{\mathrm{a}}$ & $0 / 5$ & $18.3 \%$ \\
\hline & Samadder (2013) [30] & + & + & + & + & + & & & & & & & & $3 / 5$ & $1-2 / 5^{\mathrm{a}}$ & $0 / 5$ & $29.7 \%$ \\
\hline & Simons (2013) [31] & + & + & + & + & + & & & & & & & & $3 / 5$ & $0-2 / 5$ & & Not report \\
\hline & Cleven (2014) [32] & + & + & + & + & + & & & & & & & & $3 / 5$ & $0-2 / 5$ & & Not report \\
\hline \multirow[t]{5}{*}{ D 3} & Bae (2011) [23] & + & + & + & + & + & + & + & + & & & & & $5 / 8$ & $0-4 / 8$ & & $32.0 \%$ \\
\hline & Rhee (2012) [26] & + & + & + & + & + & + & + & + & & & & & $5 / 8$ & $1-4 / 8^{a}$ & $0 / 8$ & $30.0 \%$ \\
\hline & Bae (2013) [28] & + & + & + & + & + & + & + & + & & & & & $5 / 8$ & $1-4 / 8^{a}$ & $0 / 8$ & $6.4 \%$ \\
\hline & Kim (2013) [29] & + & + & + & + & + & + & + & + & & & & & $5 / 8$ & $1-4 / 8^{\mathrm{a}}$ & $0 / 8$ & $29.1 \%$ \\
\hline & $\operatorname{Kim}(2009)^{\mathrm{b}}[18]$ & + & + & + & + & + & + & + & + & & & & & $5 / 8$ & $1-4 / 8$ & $0 / 8$ & $11.6 \%$ \\
\hline \multirow[t]{4}{*}{ D 4} & $\operatorname{Kim}(2009)^{c}[18]$ & + & + & + & + & + & + & + & + & & & & & $6 / 8$ & $1-5 / 8$ & $0 / 8$ & $7.5 \%$ \\
\hline & Ogino (2009) [19] & + & + & + & + & + & + & + & + & & & & & $6 / 8$ & $1-5 / 8$ & $0 / 8$ & $19.4 \%$ \\
\hline & Dahlin (2010) [8] & + & + & + & + & + & + & + & + & & & & & $6 / 8$ & $1-5 / 8$ & $0 / 8$ & $14.2 \% 11.4 \%^{\mathrm{d}}$ \\
\hline & Dahlin (2011) [24] & + & + & + & + & + & + & + & + & & & & & $6 / 8$ & $1-5 / 8$ & $0 / 8$ & $12.3 \%$ \\
\hline D 5 & Rijnsoever (2002) [12] & & & & & & & & + & & + & & MDR1 & $2 / 3$ & $0-1 / 3$ & & $32.0 \%$ \\
\hline D 6 & Ward (2003) [37] & & & & & & & & + & + & + & + & MINT12 & $4 / 5$ & $0-3 / 5$ & & $15.4 \%$ \\
\hline \multirow[t]{2}{*}{ D 7} & Kakar (2008) [15] & & & & & & & + & + & + & & + & RASSF2, ID4, HIC & $3 / 7$ & $0-2 / 7$ & & $23.2 \%$ \\
\hline & Kakar (2012) [39] & & & & & & & + & + & + & & + & RASSF2, ID4, HIC & $3 / 7$ & $0-2 / 7$ & & $48.5 \%$ \\
\hline D 8 & Jover (2011) [5] & + & & + & + & + & & + & & & & & & $3 / 5$ & $0-2 / 5$ & & $29.5 \%$ \\
\hline D 9 & Hokazono (2014) [33] & + & & & & & & & + & & & & $\begin{array}{l}\text { ID4, MGMT, TIMP3, TSP1, } \\
\text { CDH13, HCAD, GATA5, } \\
\text { RSASF1A, HLTF, HRK, } \\
\text { KIRREL2, SLC13A5, TSLC1 }\end{array}$ & $7 / 15$ & $1-6 / 15$ & $0 / 15$ & $18.3 \%$ \\
\hline D 10 & Wang (2014) [36] & + & & & & & & + & + & + & & & MGMT, P14ARF & $3 / 5$ & $0-2 / 5$ & & $24.0 \%$ \\
\hline D 11 & Barault (2008) [14] & & & & & & & + & + & + & + & + & & $4 / 5$ & $1-3 / 5$ & $0 / 5$ & $16.7 \%$ \\
\hline
\end{tabular}

D 11 Barault (2008) [14] 
Table 1 CIMP definitions and prevalence in studies on colorectal cancer prognosis (Continued)

\begin{tabular}{|c|c|c|c|c|c|c|c|c|c|c|c|c|c|c|c|c|c|}
\hline D 12 & Yagi (2010) [22] & + & + & + & + & + & & + & + & + & + & + & MINT17 & $6 / 11$ & $1-5 / 11$ & $0 / 11$ & $11.4 \%$ \\
\hline D 13 & Zlobec (2012) [27] & + & & + & & & + & + & + & & & & & $4 / 5$ & $1-3 / 5$ & $0 / 5$ & $7.1 \%$ \\
\hline D 14 & Li (2014) [34] & & & & + & & & + & + & + & & + & MGMT, APC & $4 / 7$ & $1-3 / 7$ & $0 / 7$ & $13.1 \%$ \\
\hline
\end{tabular}

${ }^{\mathrm{a} C I M P}$ was classified into three categories, but for analysis of prognosis only two categories were used (CIMP-H vs. CIMP-L/N)

${ }^{\mathrm{b}}$ CIMP classification 1 of the study. CIMP-H was defined as $\geq 5 / 8$ methylated markers, CIMP-L as $1 / 8$ to $4 / 8$ methylated markers, and CIMP-N as 0/8 methylated markers

${ }^{c}$ CIMP classification 2 of the study. CIMP-H was defined as $\geq 6 / 8$ methylated markers, CIMP-L as $1 / 8$ to $5 / 8$ methylated markers, and CIMP-N as 0/8 methylated markers

${ }^{\mathrm{d}} \mathrm{CIMP}+$ or CIMP-H prevalence is $14.2 \%$ in NSHDS study and $11.4 \%$ in CRUMS study. MSHDS and CRUMS are names of two study included in Dahlin et al. study 
Table 2 CIMP definitions and prevalence in studies on survival after specific colorectal cancer therapies according to CIMP status

\begin{tabular}{|c|c|c|c|c|c|c|c|c|c|c|c|c|c|c|c|c|c|}
\hline \multirow[t]{3}{*}{ Definition } & \multirow[t]{3}{*}{ First author (year) } & \multicolumn{11}{|c|}{ Common CIMP genes } & \multirow[t]{3}{*}{ Other CIMP genes } & \multicolumn{3}{|c|}{ CIMP category } & \multirow{3}{*}{$\begin{array}{l}\mathrm{CIMP}+/-\mathrm{H} \\
\text { prevalence }\end{array}$} \\
\hline & & \multirow[b]{2}{*}{ CACNA1G } & \multirow[b]{2}{*}{ IGF2 } & \multirow[b]{2}{*}{ NEUROG1 } & \multirow[b]{2}{*}{ RUNX3 } & \multirow[b]{2}{*}{ SOCS1 } & \multirow[b]{2}{*}{ CRABP1 } & \multirow[b]{2}{*}{ MLH1 } & \multirow[b]{2}{*}{ p16 } & \multirow[b]{2}{*}{ MINT1 } & \multirow[b]{2}{*}{ MINT2 } & \multirow[b]{2}{*}{ MINT31 } & & \multirow{2}{*}{$\begin{array}{l}\text { CIMP+ } \\
\text { CIMP-H }\end{array}$} & \multicolumn{2}{|l|}{ CIMP- } & \\
\hline & & & & & & & & & & & & & & & CIMP-L & CIMP-N & \\
\hline \multirow[t]{4}{*}{$\mathrm{D} 2$} & $\operatorname{Min}(2011)$ [25] & + & + & + & + & + & & & & & & & & $3 / 5$ & $1-2 / 5$ & $0 / 5$ & $13.9 \%$ \\
\hline & Donada (2013) [40] & + & + & + & + & + & & & & & & & & $3 / 5$ & $1-2 / 5$ & $0 / 5$ & $18.3 \%$ \\
\hline & Jo (2012) [44] & + & + & + & + & + & & & & & & & & $3 / 5$ & $0-2 / 5$ & & $10.0 \%$ \\
\hline & Shiovitz (2014) [35] & + & + & + & + & + & & & & & & & & $3 / 5$ & $0-2 / 5$ & & $23.6 \%$ \\
\hline D 3 & Han (2013) [45] & + & + & + & + & + & + & + & + & & & & & $5 / 8$ & $1-4 / 8$ & $0 / 8$ & $7.8 \%$ \\
\hline D 5 & Rijinsoever (2003) [41] & & & & & & & & + & & + & & MDR1 & $2 / 3$ & $0-1 / 3$ & & $32.5 \%$ \\
\hline D 8 & Jover (2011) [5] & + & & + & + & + & & + & & & & & & $3 / 5$ & $0-2 / 5$ & & $29.5 \%$ \\
\hline D 10 & Wang (2014) [36] & & & & & & & + & + & + & & & MGMT, P14 ${ }^{\mathrm{ARF}}$ & $3 / 5$ & $<3 / 5$ & & $24.0 \%$ \\
\hline D 14 & Li (2014) [34] & & & & + & & & + & + & + & & + & MGMT, APC & $4 / 7$ & $1-3 / 7$ & $0 / 7$ & $13.1 \%$ \\
\hline \multirow[t]{2}{*}{ D 15} & Ogino (2007) [42] & + & + & + & + & + & + & + & + & + & & + & MGMT, IGFBP3, WRN & $9 / 13^{a}$ & $1-8 / 13$ & 0/13 & $10.0 \%$ \\
\hline & & & & & & & & & & & & & & $7 / 13^{b}$ & $1-6 / 13$ & $0 / 13$ & $16.7 \%$ \\
\hline D 16 & Shen (2007) [43] & & & & & & & & + & + & & + & $\mathrm{P} 14^{\mathrm{ARF}}$ & $2 / 4$ & $0-1 / 4$ & & $15.4 \%$ \\
\hline
\end{tabular}

${ }^{a}$ CIMP classification 1 of the study. CIMP-H was defined as $\geq 9 / 13$ methylated markers, CIMP-L as 1/13 to 8/13 methylated markers and CIMP-N as 0/13 methylated markers

${ }^{b}$ CIMP classification 2 of the study. CIMP-H was defined as $\geq 7 / 13$ methylated markers, CIMP-L as $1 / 13$ to 6/13 methylated markers and CIMP-N as 0/13 methylated markers 
Table 3 Overall survival among patients with colorectal cancer according to CIMP status

\begin{tabular}{|c|c|c|c|c|c|c|c|c|c|c|}
\hline \multirow[t]{2}{*}{ CIMP definition } & \multirow[t]{2}{*}{ First author (year) } & \multirow[t]{2}{*}{ Subgroup } & \multirow{2}{*}{$\begin{array}{l}\text { Subgroup } \\
\text { size }\end{array}$} & \multirow[t]{2}{*}{ Analysis } & \multirow[t]{2}{*}{ Survival } & \multicolumn{2}{|l|}{ HR $(95 \% \mathrm{Cl})$} & \multirow{2}{*}{$\begin{array}{l}\text { Comparison } \\
\text { group }\end{array}$} & \multirow[t]{2}{*}{$p$ value } & \multirow[t]{2}{*}{ Covariates adjusted for } \\
\hline & & & & & & $\mathrm{CIMP}+/ \mathrm{CIMP}-\mathrm{H}$ & CIMP-L & & & \\
\hline \multicolumn{11}{|l|}{ CRC patients } \\
\hline D 1 & Lee (2008) [16] & All & 134 & U & OS & $1.59(0.87-2.88)$ & & CIMP- & 0.13 & No \\
\hline D 2 & Sanchez (2009) [21] & All & 391 & M & OS & $1.56(0.88-2.78)^{\mathrm{a}}$ & & CIMP- & 0.13 & Age, sex, stage, location, $\mathrm{MSI}^{\mathrm{b}}$ \\
\hline D 6 & Ward (2003) [37] & All & 609 & $U$ & OS & $1.30(0.70-2.20)$ & & CIMP- & 0.37 & No \\
\hline D 7 & Kakar (2012) [39] & All & 33 & $U$ & OS & $1.19(0.51-2.58)$ & & CIMP- & 0.69 & No \\
\hline D 14 & Li (2014) [34] & All & 282 & M & OS & $2.31(1.02-5.24)$ & & CIMP- & 0.04 & Age, stage, location, differentiation \\
\hline D 2 & Samadder (2013) [30] & All & 563 & M & OS & $1.12(0.81-1.55)$ & $0.86(0.60-1.23)$ & CIMP-N & 0.60 & Age, stage, grade, location ${ }^{c}$ \\
\hline D 3 & Kim (2009) [18] & All & 320 & $U$ & OS & $1.81(0.91-3.66)$ & $1.33(0.78-2.27)$ & CIMP-N & 0.24 & No \\
\hline D 4 & Kim (2009) [18] & All & 320 & U & OS & $2.46(1.16-5.19)$ & $1.30(0.76-2.22)$ & CIMP-N & 0.05 & No \\
\hline D 14 & Li (2014) [34] & All & 282 & M & OS & $3.06(1.19-7.89)$ & $0.95(0.60-1.52)$ & CIMP-N & 0.02 & Age, stage, location, differentiation \\
\hline \multicolumn{11}{|c|}{ CRC patients by cancer stages } \\
\hline D 14 & Li (2014) [34] & I-III & 149 & M & OS & $0.52(0.12-2.22)$ & & CIMP- & 0.38 & Age, stage, location, differentiation \\
\hline D 6 & Ward (2003) [37] & I-III & 476 & $U$ & OS & $1.20(0.60-2.80)$ & & CIMP- & 0.60 & No \\
\hline D 11 & Barault (2008) [14] & I-II, colon, MSS & 246 & M & $\mathrm{OS}^{\mathrm{d}}$ & $2.90(1.53-5.49)$ & $1.85(1.37-2.51)$ & CIMP-N & $<0.01$ & Age, stage, BRAF, KRAS \\
\hline D 2 & Donada (2013) [40] & II, colon & 120 & M & OS & 0.60 & & CIMP- & 0.30 & All variables ${ }^{e}$ \\
\hline D 14 & Li (2014) [34] & III-IV & 129 & M & OS & $1.75(0.95-3.23)$ & & CIMP- & 0.07 & Age, stage, location, differentiation \\
\hline \multicolumn{11}{|c|}{ CRC patients by location of CRC } \\
\hline D 3 & Bae (2013) [28] & Proximal & 165 & M & OS & $0.84(0.42-1.69)$ & & CIMP- & 0.62 & Stage' differentiation $^{f}$ \\
\hline D 3 & Bae (2013) [28] & Distal & 327 & M & OS & $1.35(0.47-3.90)$ & & CIMP- & 0.58 & Stage' differentiation ${ }^{f}$ \\
\hline D 4 & Ogino (2009) [19] & Colon & 649 & M & OS & $0.78(0.47-1.29)$ & $1.01(0.77-1.33)$ & CIMP-N & & Age, sex, stage, BRAF, KRAS, MSI9 \\
\hline D 2 & Donada (2013) [40] & Colon, II & 120 & M & OS & 0.60 & & CIMP- & 0.30 & All variables ${ }^{e}$ \\
\hline D 1 & Samowitz (2005) [13] & Colon, MSS & 803 & M & OS & $0.88(0.66-1.18)$ & & CIMP- & & Age, stage, location, BRAF \\
\hline D 11 & Barault (2008) [14] & Colon, MSS, I-II & 246 & M & $\mathrm{OS}^{\mathrm{d}}$ & $2.90(1.53-5.49)$ & $1.85(1.37-2.51)$ & CIMP- & $<0.01$ & Age, stage, BRAF, KRAS \\
\hline D 3 & Bae (2013) [28] & Rectal & 242 & M & OS & $4.13(1.27-13.46)$ & & CIMP- & 0.02 & Stage' differentiation $^{f}$ \\
\hline \multicolumn{11}{|c|}{ CRC patients by microsatellite instability } \\
\hline D 1 & Lee (2008) [16] & MSS & 115 & $U$ & OS & $1.96(1.06-3.61)$ & & CIMP- & 0.03 & No \\
\hline D 6 & Ward (2003) [37] & MSS & 547 & M & OS & $2.10(1.10-4.00)$ & & CIMP- & 0.02 & Stage, vascular space invasion \\
\hline D 7 & Kakar (2008) [15] & MSS & 69 & M & OS & $0.86(0.35-2.13)$ & & CIMP- & 0.70 & Age, sex, stage, LOH, BRAF \\
\hline D 6 & Ward (2003) [37] & MSS, I-III & 464 & M & OS & $2.40(0.94-6.00)$ & & CIMP- & 0.06 & Stage, vascular space invasion \\
\hline D 1 & Samowitz (2005) [13] & MSS, colon & 803 & M & OS & $0.88(0.66-1.18)$ & & CIMP- & & Age, stage, location, BRAF \\
\hline D 11 & Barault (2008) [14] & MSS, colon, I-II & 246 & M & $\mathrm{OS}^{\mathrm{d}}$ & $2.90(1.53-5.49)$ & $1.85(1.37-2.51)$ & CIMP-N & $<0.01$ & Age, stage, BRAF, KRAS \\
\hline
\end{tabular}


Table 3 Overall survival among patients with colorectal cancer according to CIMP status (Continued)

\begin{tabular}{|c|c|c|c|c|c|c|c|c|c|}
\hline \\
\hline D 3 & Bae (2011) [23] & \multicolumn{8}{|c|}{ CRC patients by microsatellite instability } \\
\hline D 3 & Rhee (2012) [26] & MSI & 207 & M & OS & $3.05(1.07-8.73)$ & CIMP- & 0.04 & Age, stage, location, BRAF/KRAS \\
\hline
\end{tabular}

$C /$ confidence interval, $H R$ hazard ratio, $M$ multivariate analysis, $O S$ overall survival, $U$ univariate analysis

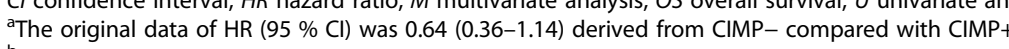

bOther covariates included in the multivariate analysis are differentiation and chemotherapy

Other covariates included in the multivariate analysis are chemotherapy and radiation therapy

${ }^{\mathrm{d}}$ Relative survival ratio of observed survival rate to the expected survival rate in a population with similar sex and age distribution derived from local mortality

eOriginal paper mentioned the multivariate analysis including all clinical, pathological, and molecular variables

fOther covariates included in the multivariate analysis are adjuvant chemotherapy

${ }^{9}$ Other covariates included in the multivariate analysis are year of diagnosis, location, and tumor grade

hOther covariates included in the multivariate analysis are Crohn's-like lymphoid reaction, peritumoral lymphocytic reaction, and postoperative chemotherapy

'Other covariates included in the multivariate analysis are grade, gross type and Crohn's-like lymphoid reaction, and peritumoral lymphocytic reaction 
Table 4 Disease-specific survival among patients with colorectal cancer according to CIMP status

\begin{tabular}{|c|c|c|c|c|c|c|c|c|c|c|}
\hline \multirow[t]{2}{*}{ CIMP definition } & \multirow[t]{2}{*}{ First author (year) } & \multirow[t]{2}{*}{ Subgroup } & \multirow{2}{*}{$\begin{array}{l}\text { Subgroup } \\
\text { size }\end{array}$} & \multirow[t]{2}{*}{ Analysis } & \multirow[t]{2}{*}{ Survival } & \multicolumn{2}{|l|}{$\mathrm{HR}(95 \% \mathrm{Cl})$} & \multirow{2}{*}{$\begin{array}{l}\text { Comparison } \\
\text { group }\end{array}$} & \multirow[t]{2}{*}{$p$ value } & \multirow[t]{2}{*}{ Covariates adjusted for } \\
\hline & & & & & & $\mathrm{CIMP}+/ \mathrm{CIMP}-\mathrm{H}$ & CIMP-L & & & \\
\hline \multicolumn{11}{|l|}{ CRC patients } \\
\hline D 2 & Simons (2013) [31] & All & 27 & M & DSS & $3.67(1.70-7.91)^{\mathrm{a}}$ & & CIMP- & & Age, sex, stage, location ${ }^{b}$ \\
\hline D 2 & Samadder (2013) [30] & All & 563 & M & DSS & $1.06(0.65-1.35)$ & $1.19(0.72-1.97)$ & CIMP-N & 0.74 & Age, stage, grade, location ${ }^{c}$ \\
\hline D 4 & Dahlin (2010) [8] & All (NSHDS) & 190 & M & DSS & $1.84(0.87-3.89)$ & $2.01(1.20-3.37)$ & CIMP-N & & Age, sex, stage, location ${ }^{d}$ \\
\hline D 4 & Dahlin (2010) [8] & All (CRUMS) & 414 & M & DSS & $1.10(0.59-2.03)$ & $1.48(1.00-2.22)$ & CIMP-N & & Age, sex, stage, location ${ }^{d}$ \\
\hline D 4 & Dahlin (2011) [24] & All & 484 & M & DSS & $1.09(0.59-2.03)$ & $1.47(0.98-2.20)$ & CIMP-N & & Age, sex, stage, location ${ }^{d}$ \\
\hline \multicolumn{11}{|c|}{ CRC patients by microsatellite instability } \\
\hline D 1 & Samowitz (2005) [13] & MSS, colon & 803 & M & DSS & $0.97(0.70-1.36)$ & & CIMP- & & Age, stage, location, BRAF \\
\hline D 4 & Dahlin (2010) [8] & MSS (NSHDS) & 166 & M & DSS & $3.05(1.40-6.63)$ & $1.89(1.12-3.21)$ & CIMP-N & & Age, sex, stage, location ${ }^{d}$ \\
\hline D 4 & Dahlin (2010) [8] & MSS (CRUMS) & 338 & M & DSS & $1.38(0.62-3.07)$ & $1.45(0.95-2.23)$ & CIMP-N & & Age, sex, stage, location ${ }^{d}$ \\
\hline D 4 & Dahlin (2010) [8] & MSI (CRUMS) & 62 & M & DSS & $1.23(0.13-11.23)$ & $3.87(0.46-32.39)$ & CIMP-N & & Age, sex, stage, location ${ }^{d}$ \\
\hline \multicolumn{11}{|c|}{ CRC patients by location of CRC } \\
\hline D 4 & Ogino (2009) [19] & Colon & 649 & M & DSS & $0.44(0.22-0.88)$ & $0.78(0.54-1.11)$ & CIMP-N & & Age, stage, BRAF, KRAS, MSI ${ }^{e}$ \\
\hline D 1 & Samowitz (2005) [13] & MSS, colon & 803 & M & DSS & $0.97(0.70-1.36)$ & & CIMP- & & Age, stage, location, BRAF \\
\hline
\end{tabular}

CI confidence interval, CRUMS and NSHDS are the names of two studies included in Dahlin et al. study, DSS disease-specific survival, $H R$ hazard ratio, $M$ multivariate analysis, $U$ univariate analysis

a Data come from early follow-up ( $\leq 2$ year). Data of late follow-up (>2 year) was 1.41 (0.43-4.57)

bother covariates included in the multivariate analysis are differentiation grade and initial adjuvant therapy

cOther covariates included in the multivariate analysis are chemotherapy and radiation therapy

dOther covariate included in the multivariate analysis is adjuvant chemotherapy

ether covariates included in the multivariate analysis are year of diagnosis, sex, site, and tumor grade 
Table 5 Disease-free survival or recurrence-free survival among patients with colorectal cancer according to CIMP status

\begin{tabular}{|c|c|c|c|c|c|c|c|c|c|c|}
\hline \multirow[t]{2}{*}{ CIMP definition } & \multirow[t]{2}{*}{ First author (year) } & \multirow[t]{2}{*}{ Subgroup } & \multirow{2}{*}{$\begin{array}{l}\text { Subgroup } \\
\text { size }\end{array}$} & \multirow[t]{2}{*}{ Analysis } & \multirow[t]{2}{*}{ Survival } & \multicolumn{2}{|l|}{$\mathrm{HR}(95 \% \mathrm{Cl})$} & \multirow{2}{*}{$\begin{array}{l}\text { Comparison } \\
\text { group }\end{array}$} & \multirow[t]{2}{*}{$p$ value } & \multirow[t]{2}{*}{ Covariates adjusted for } \\
\hline & & & & & & $\mathrm{CIMP}+/ \mathrm{CIMP}-\mathrm{H}$ & CIMP-L & & & \\
\hline \multicolumn{11}{|l|}{ CRC patients } \\
\hline D 2 & Kalady (2009) [17] & All & 357 & M & RFS & $2.08(0.65-6.65)^{\mathrm{a}}$ & & CIMP- & 0.21 & Age, sex, stage, MSI ${ }^{\mathrm{b}}$ \\
\hline D 9 & Hokazono (2014) [33] & All & 104 & M & RFS & $0.29(0.02-1.42)$ & & CIMP- & 0.15 & Not report \\
\hline \multicolumn{11}{|c|}{ CRC patients by cancer stages } \\
\hline D 9 & Hokazono (2014) [33] & $|-| \mid$ & 50 & M & RFS & $0.01(0.00-2.28)$ & & CIMP- & & Not report \\
\hline D 1 & Ju (2011) [38] & $|-|||$ & 53 & M & RFS & $1.05(0.33-3.39)$ & & CIMP- & 0.93 & Age, sex, stage \\
\hline D 2 & $\operatorname{Min}(2011)[25]$ & $|-|||$ & 124 & M & RFS & $0.81(0.21-3.14)$ & & CIMP- & & Age, sex, stage, BRAF, KRAS, MGMT' \\
\hline D 6 & Ward (2003) [37] & $|-|||$ & 476 & U & RFS & $0.90(0.50-2.00)$ & & CIMP- & 0.94 & No \\
\hline D 8 & Jover (2011) [5] & $\|-I\|$ & 196 & M & DFS & $1.20(0.80-2.00)$ & & CIMP- & 0.40 & Age, stage, adjuvant chemotherapy \\
\hline D 10 & Wang (2014) [36] & $\|-\| \mid$ & 50 & M & DFS & $2.94(1.19-7.22)$ & & CIMP- & 0.02 & Stage, location \\
\hline D 2 & Donada (2013) [40] & II, colon & 120 & M & DFS & 0.58 & & CIMP- & 0.20 & All variables $^{c}$ \\
\hline D 9 & Hokazono (2014) [33] & III & 39 & M & RFS & $0.45(0.01-2.23)$ & & CIMP- & & Not report \\
\hline \multicolumn{11}{|c|}{ CRC patients by location of CRC } \\
\hline D 3 & Bae (2013) [28] & Proximal & 165 & M & DFS & $1.00(0.53-1.88)$ & & CIMP- & 0.99 & Stage $^{c}$ \\
\hline D 3 & Bae (2013) [28] & Distal & 327 & M & DFS & $1.31(0.51-3.36)$ & & CIMP- & 0.58 & Stage $^{c}$ \\
\hline D 2 & Donada (2013) [40] & Colon, II & 120 & M & DFS & 0.58 & & CIMP- & 0.20 & All variables $^{c}$ \\
\hline D 1 & Samowitz (2009) [20] & Rectal & 990 & M & DFS & $1.32(0.88-1.97)$ & & CIMP- & & Age, stage, BRAF, MSI, KRAS, TP53 ${ }^{\mathrm{d}}$ \\
\hline D 3 & Bae (2013) [28] & Rectal & 242 & M & DFS & $2.90(1.04-8.08)$ & & CIMP- & 0.04 & Stage $^{c}$ \\
\hline \multicolumn{11}{|c|}{ CRC patients by microsatellite instability } \\
\hline D 3 & $\operatorname{Kim}(2013)$ [29] & MSI & 220 & M & DFS & $2.25(1.11-4.57)$ & & CIMP- & 0.03 & Age, stage, differentiation, BRAF \\
\hline
\end{tabular}

$C l$ confidence interval, $D F S$ disease-free survival, $H R$ hazard ratio, $M$ multivariate analysis, $R F S$ recurrence-free survival, $U$ univariate analysis

aThe original data of HR $(95 \% \mathrm{Cl})$ was 0.48 (0.15-1.53) derived from CIMP- compared with CIMP+

OOther covariates included in the multivariate analysis are: differentiation, chemotherapy

Criginal paper mentioned the multivariate analysis including all clinical, pathological and molecular variables

${ }^{\mathrm{d}}$ Multivariate analysis adjusted BRAF, MSI, KRAS, and TP53, in addition to age and stage, the result adjusted for only age and stage was 1.45 (1.02-2.07)

'Other covariates included in the multivariate analysis are Crohn's-like lymphoid reaction and peritumoral lymphocytic reaction 
Table 6 Survival of colorectal cancer patients treated with surgery and chemotherapy compared with patients treated with surgery alone

\begin{tabular}{llllllll}
\hline CIMP group & CIMP definition & Study & Group size & Analysis & Survival & HR (95 \% Cl) & $p$ value \\
\hline CIMP+ & D 14 & Li (2014) [34] & 37 & M & OS & $0.71(0.20-2.54)$ & 0.60 \\
& D 2 & Shiovitz (2014) $)^{\mathrm{a}}[35]$ & 145 & M & OS & $0.62(0.37-1.05)^{\mathrm{b}}$ & 0.07 \\
& D 8 & Jover (2011) [5] & 89 & M & DFS & $0.80(0.30-2.00)$ & 0.60 \\
CIMP- & D 14 & Li (2014) [34] & 245 & M & OS & $1.09(0.65-1.82)$ & 0.75 \\
& D 2 & Shiovitz (2014) $[35]$ & 470 & M & OS & $1.38(1.00-1.89)$ & 0.05 \\
& D 5 & Rijinsoever (2003) [41] & 139 & M & DSS & $0.96(0.62-1.49)$ & 0.86 \\
& D 8 & Jover (2011) [5] & 213 & M & DFS & $0.40(0.20-0.60)$ & $<0.01$ \\
\hline
\end{tabular}

Cl confidence interval, HR hazard ratio

${ }^{a}$ Results comparing patients treated with surgery + LV + FU + IFL with patients treated with surgery + LV + FU

${ }^{\mathrm{b}} \mathrm{HR}$ of surgery + chemotherapy compared with surgery alone in CIMP+ group was not reported directly in the study and was calculated according to $95 \% \mathrm{Cl}$

the most commonly used (Table 1). Other genes like MGMT and ID4 were only included by a few studies.

Furthermore, categorization of CIMP in two categories (CIMP+ vs. CIMP-) or three categories (CIMP-H vs. CIMP-L or CIMP-0) may result in different associations with survival after CRC. CIMP-L represents a subclass of CRCs with intermediate methylation level and this intermediate methylation subclass shows distinct features compared with CIMP-H or CIMP-0, respectively. CIMP-H CRC shows associations with MSI, more frequent BRAF mutation, and less KRAS and TP53 mutations, whereas CIMP-L CRCs are associated with KRAS mutation. CIMP-0 is characterized by frequent TP53 mutation [22, 50-52]. Another source of heterogeneity is threshold for CIMP-H. In the study by Kim et al. [18], CIMP-H defined by six or more methylated loci (definition 4) showed a statistically significant and a somewhat stronger association with poorer survival compared to a cutoff of five or more methylated loci (definition 3). Lee and colleagues also found that higher cutoff for CIMP+ could better separate the survival curve of CIMP+ and CIMP- patients among MSI CRC [53].

Also, the use of different laboratory methods and criteria for threshold of methylation may influence findings. MSP is a simple, sensitive, and specific method for determining the methylation status of virtually any CpGrich region [46]. MethyLight, compared with MSP, is not only a sensitive and accurate method to detect methylation but also a quantitative real-time PCR analysis technique with high-throughput capability [47]. Quantitative DNA methylation analysis is used because it was found that low levels of DNA methylation do not generally silence gene expression $[54,55]$. Moreover, the cutoff for gene methylation used in each method also varied between studies. Besides, DNA samples obtained from tissue preserved in different ways might cause inconsistent methylation levels. A previous study have shown that DNA samples obtained from FFPE tissue can provide accurate and reproducible results in DNA methylation analyses by using Infinium HumanMethylation450 BeadChip (HM-450 K) assays or nested MSP [56]. However, there is limited evidence on the effect of different laboratory methods and no obvious differences in outcomes according to laboratory method were observed in this review with respect to the prognostic value of CIMP. Further research should address the potential role of laboratory methods for the prognostic value of CIMP definition in more detail.

Besides the difference in CIMP definition and laboratory methods, failure in controlling important confounders in the respective studies may cause the observed discrepancies in the CIMP literature. Patient and clinical factors that are associated with both CIMP and CRC prognosis, such as age, tumor location, and stage, were adjusted for in most but not in all of the included studies. CIMP-high tumors are more often present in women than in men. However, gender was only adjusted for in a few studies and none of the included studies reported prognostic implications of CIMP according to gender.

Patients with MSI CRC were shown to have better prognosis than patients with microsatellite stability (MSS) CRC [17]. Ward et al. [37] even found that the adverse prognostic effect of CIMP could be reversed by MSI. However, in studies by Bea et al. and by Kim et al. [23, 29], CIMP+ was found to be associated with poorer survival among patients with MSI CRC. In addition, BRAF mutation was shown to be a predictor of poorer outcome in patients with MSS CRC [15]. In the study by Samowitz et al. [13], the association between CIMP+ and worse outcomes of MSS CRC disappeared after adjusting for BRAF. The impact of other genetic factors, such as KRAS mutation, on the prognosis of CRC is also not consistent [20, 21, 29]. Sporadic MSI-H and BRAF mutations frequently occur in a subset of CIMP tumors; however, they were only assessed in a few studies but may play a role in the association between CIMP and CRC prognosis. Some researchers even suggested new major subtypes of CRC that combine two or even four of these factors which 
might provide a new approach to investigate and understand the relationship between genetic markers and the prognosis of CRC [57].

Results regarding to potential role of CIMP in predicting success of specific therapies were inconsistent. In one study [35], addition of IFL to therapy with surgery + $\mathrm{LV}+\mathrm{FU}$ was associated with tentatively enhanced survival among CIMP+ patients and tentatively worse survival among CIMP- patients. More and larger studies are needed to find out if CIMP is a relevant molecular biomarker for adjuvant therapy decision-making in CRC.

The main limitation of our review was the lack of studies investigating the same CIMP definitions. Moreover, the sample sizes of included studies were all less than 1000 and mostly less than 400 cases with four of the studies only including less than 100 cases. The statistical power in these studies became even weaker when analyzing subgroups. Thus, more studies in larger patient populations are needed.

\section{Conclusions}

In summary, no clear differences could be observed regarding the association of CIMP and CRC prognosis that are based on the different CIMP definitions due to the high heterogeneity of CIMP definitions, subgroups, and outcomes investigated. Although CIMP marker panel, CIMP grouping, and threshold of CIMP+ or CIMP-H may have potential impact on the prognostic value of CIMP among CRC patients, evidence on their specific impact is still limited. Comparative analyses of different CIMP panels in the same large study populations are needed to further clarify the role of CIMP definitions and to find out how methylation information can best be used to predict CRC prognosis and response to specific CRC therapies.

\section{Additional files}

Additional file 1: Table S1. General information of studies investigating survival after colorectal cancer according to CIMP status. (DOCX 28 kb)

Additional file 2: Table S2. General information of studies investigating therapy response after colorectal cancer according to CIMP status. (DOCX $22 \mathrm{~kb}$ )

Additional file 3: Table S3. Method used for methylation analysis in studies on colorectal cancer survival according to CIMP status. (DOCX 24 kb)

Additional file 4: Table S4. Method used for methylation analysis in studies investigating therapy response after colorectal according to CIMP status. (DOCX $20 \mathrm{~kb}$ )

Additional file 5: Table S5. Results of studies on survival after colorectal cancer according to CIMP status reporting a $p$ value only. (DOCX $22 \mathrm{~kb}$ )

\section{Abbreviations}

COBRA: combined bisulfite restriction analysis; $\mathrm{Cl}$ : confidence interval; CIMP: CPG island methylator phenotype; CIMP+: CIMP-positive; CIMP-: CIMP-negative; CIMP-H: CIMP-high; CIMP-L: CIMP-low;

CIMP-N: CIMP-negative; CRC: colorectal cancer; DFS: disease-free survival;
DSS: disease-specific survival; FFPE: formalin-fixed and paraffin-embedded; FF: fresh-frozen; HR: hazard ratio; M: multivariate analysis; MSHRM: methylation-sensitive high-resolution melting; MSI: microsatellite instability; MSP: methylation-specific PCR; MSS: microsatellite stability;

OS: overall survival; RFS: recurrence-free survival.

\section{Competing interests}

The authors declare that they have no competing interests.

\section{Authors' contributions}

$H B, M H$, and $M J$ designed the study. $M J$ and $X G$ extracted the data. MJ wrote the manuscript. $\mathrm{HB}, \mathrm{MH}$, and $\mathrm{YZ}$ revised the manuscript. All authors read and approved the final manuscript.

\section{Acknowledgements}

This study was supported in part by the China Scholarship Council (CSC). The sponsor had no role in the study design and in the collection, analysis, and interpretation of data.

Received: 9 December 2015 Accepted: 23 February 2016

Published online: 02 March 2016

\section{References}

1. Ferlay J, Soerjomataram I, Dikshit R, Eser S, Mathers C, Rebelo M, et al. Cancer incidence and mortality worldwide: sources, methods and major patterns in GLOBOCAN 2012. Int J Cancer. 2015;136(5):E359-86. doi:10.1002/ijc.29210.

2. Siegel R, Desantis C, Jemal A. Colorectal cancer statistics, 2014. CA Cancer J Clin. 2014;64(2):104-17. doi:10.3322/caac.21220.

3. Toyota M, Ahuja N, Ohe-Toyota M, Herman JG, Baylin SB, Issa JP. CpG island methylator phenotype in colorectal cancer. Proc Natl Acad Sci U S A. 1999;96(15):8681-6.

4. Jover R, Nguyen TP, Perez-Carbonell L, Zapater P, Paya A, Alenda C, et al. 5-Fluorouracil adjuvant chemotherapy does not increase survival in patients with CpG island methylator phenotype colorectal cancer. Gastroenterology. 2011;140(4):1174-81. doi:10.1053/j.gastro.2010.12.035.

5. Kim YS, Deng G. Epigenetic changes (aberrant DNA methylation) in colorectal neoplasia. Gut Liver. 2007;1(1):1-11. doi:10.5009/gnl.2007.1.1.1.

6. Herman JG, Baylin SB. Gene silencing in cancer in association with promoter hypermethylation. N Engl J Med. 2003;349(21):2042-54. doi:10.1056/NEJMra023075.

7. Nagasaka T, Koi M, Kloor M, Gebert J, Vilkin A, Nishida N, et al. Mutations in both KRAS and BRAF may contribute to the methylator phenotype in colon cancer. Gastroenterology. 2008;134(7):1950-60. doi:10.1053/j.gastro.2008.02.094. 60.e1.

8. Dahlin AM, Palmqvist R, Henriksson ML, Jacobsson M, Eklof V, Rutegard J, et al. The role of the $\mathrm{CpG}$ island methylator phenotype in colorectal cancer prognosis depends on microsatellite instability screening status. Clin Cancer Res. 2010;16(6):1845-55. doi:10.1158/1078-0432.ccr-09-2594.

9. Weisenberger DJ, Siegmund KD, Campan M, Young J, Long TI, Faasse MA, et al. CpG island methylator phenotype underlies sporadic microsatellite instability and is tightly associated with BRAF mutation in colorectal cancer. Nat Genet. 2006;38(7):787-93. doi:10.1038/ng1834.

10. Juo YY, Johnston FM, Zhang DY, Juo HH, Wang H, Pappou EP, et al. Prognostic value of $\mathrm{CpG}$ island methylator phenotype among colorectal cancer patients: a systematic review and meta-analysis. Ann Oncol. 2014;25(12):2314-27. doi:10.1093/annonc/mdu149.

11. Liberati A, Altman DG, Tetzlaff J, Mulrow C, Gotzsche PC, loannidis JP, et al. The PRISMA statement for reporting systematic reviews and meta-analyses of studies that evaluate health care interventions: explanation and elaboration. PLoS Med. 2009;6(7):e1000100. doi:10.1371/journal.pmed.1000100.

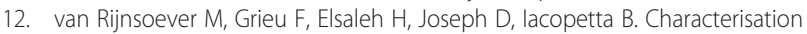
of colorectal cancers showing hypermethylation at multiple CpG islands. Gut. 2002;51(6):797-802.

13. Samowitz WS, Sweeney C, Herrick J, Albertsen H, Levin TR, Murtaugh MA, et al. Poor survival associated with the BRAF V600E mutation in microsatellitestable colon cancers. Cancer Res. 2005;65(14):6063-70.

14. Barault L, Charon-Barra C, Jooste V, de la Vega MF, Martin L, Roignot P, et al. Hypermethylator phenotype in sporadic colon cancer: study on a population-based series of 582 cases. Cancer Res. 2008;68(20):8541-6. doi:10.1158/0008-5472.can-08-1171. 
15. Kakar S, Deng G, Sahai V, Matsuzaki K, Tanaka H, Miura S, et al. Clinicopathologic characteristics, CpG island methylator phenotype, and BRAF mutations in microsatellite-stable colorectal cancers without chromosomal instability. Arch Pathol Lab Med. 2008;132(6):958-64. doi:10.1043/1543-2165(2008)132[958:cccimp]2.0.co;2.

16. Lee S, Cho NY, Choi M, Yoo EJ, Kim JH, Kang GH. Clinicopathological features of $\mathrm{CpG}$ island methylator phenotype-positive colorectal cancer and its adverse prognosis in relation to KRAS/BRAF mutation. Pathol Int. 2008;58(2):104-13. doi:10.1111/j.1440-1827.2007.02197.x

17. Kalady MF, Sanchez JA, Manilich E, Hammel J, Casey G, Church JM. Divergent oncogenic changes influence survival differences between colon and rectal adenocarcinomas. Dis Colon Rectum. 2009;52(6):1039-45. doi:10.1007/DCR.0b013e31819edbd4.

18. Kim JH, Shin SH, Kwon HJ, Cho NY, Kang GH. Prognostic implications of CpG island hypermethylator phenotype in colorectal cancers. Virchows Arch. 2009;455(6):485-94. doi:10.1007/s00428-009-0857-0.

19. Ogino S, Nosho K, Kirkner GJ, Kawasaki T, Meyerhardt JA, Loda M, et al. CpG island methylator phenotype, microsatellite instability, BRAF mutation and clinical outcome in colon cancer. Gut. 2009:58(1):90-6. doi:10.1136/gut.2008.155473.

20. Samowitz WS, Curtin K, Wolff RK, Tripp SR, Caan BJ, Slattery ML. Microsatellite instability and survival in rectal cancer. Cancer Causes Control. 2009;20(9):1763-8. doi:10.1007/s10552-009-9410-3.

21. Sanchez JA, Krumroy L, Plummer S, Aung P, Merkulova A, Skacel M, et al. Genetic and epigenetic classifications define clinical phenotypes and determine patient outcomes in colorectal cancer. Br J Surg. 2009;96(10):1196-204. doi:10.1002/bjs.6683.

22. Yagi K, Akagi K, Hayashi H, Nagae G, Tsuji S, Isagawa T, et al. Three DNA methylation epigenotypes in human colorectal cancer. Clin Cancer Res. 2010;16(1):21-33. doi:10.1158/1078-0432.ccr-09-2006.

23. Bae JM, Kim MJ, Kim JH, Koh JM, Cho NY, Kim TY, et al. Differential clinicopathological features in microsatellite instability-positive colorectal cancers depending on CIMP status. Virchows Arch. 2011;459(1):55-63. doi:10.1007/s00428-011-1080-3.

24. Dahlin AM, Henriksson ML, Van Guelpen B, Stenling R, Oberg A, Rutegard J, et al. Colorectal cancer prognosis depends on T-cell infiltration and molecular characteristics of the tumor. Mod Pathol. 2011;24(5):671-82. doi:10.1038/modpathol.2010.234.

25. Min BH, Bae JM, Lee EJ, Yu HS, Kim YH, Chang DK, et al. The CpG island methylator phenotype may confer a survival benefit in patients with stage II or III colorectal carcinomas receiving fluoropyrimidine-based adjuvant chemotherapy. BMC Cancer. 2011;11:344. doi:10.1186/1471-2407-11-344.

26. Rhee Y, Kim MJ, Bae JM, Koh JM, Cho N-Y, Juhnn Y-S, et al. Clinical outcomes of patients with microsatellite-unstable colorectal carcinomas depend on L1 methylation level. Ann Surg Oncol. 2012;19(11):3441-8.

27. Zlobec I, Bihl MP, Foerster A, Rufle A, Terracciano L, Lugli A. Stratification and prognostic relevance of Jass's molecular classification of colorectal cancer. Front Oncol. 2012;2:7. doi:10.3389/fonc.2012.00007.

28. Bae JM, Kim JH, Cho NY, Kim TY, Kang GH. Prognostic implication of the $\mathrm{CpG}$ island methylator phenotype in colorectal cancers depends on tumour location. Br J Cancer. 2013;109(4):1004-12. doi:10.1038/bjc.2013.430.

29. Kim JH, Rhee YY, Bae JM, Kwon HJ, Cho NY, Kim MJ, et al. Subsets of microsatellite-unstable colorectal cancers exhibit discordance between the CpG island methylator phenotype and MLH1 methylation status. Mod Pathol. 2013;26(7):1013-22. doi:10.1038/modpathol.2012.241.

30. Samadder NJ, Vierkant RA, Tillmans LS, Wang AH, Weisenberger DJ, Laird PW, et al. Associations between colorectal cancer molecular markers and pathways with clinicopathologic features in older women. Gastroenterology. 2013;145(2):348-56. doi:10.1053/j.gastro.2013.05.001. e1-2.

31. Simons CC, Hughes LA, Smits KM, Khalid-de Bakker CA, de Bruine AP, Carvalho B, et al. A novel classification of colorectal tumors based on microsatellite instability, the CpG island methylator phenotype and chromosomal instability: implications for prognosis. Ann Oncol. 2013;24(8):2048-56. doi:10.1093/annonc/mdt076.

32. Cleven AH, Derks S, Draht MX, Smits KM, Melotte V, Van Neste L, et al. CHFR promoter methylation indicates poor prognosis in stage ॥ microsatellite stable colorectal cancer. Clin Cancer Res. 2014;20(12):3261-71. doi:10.1245/s10434-014-3888-y10.1158/1078-0432.ccr-12-3734.

33. Hokazono K, Ueki T, Nagayoshi K, Nishioka Y, Hatae T, Koga Y, et al. A $\mathrm{CpG}$ island methylator phenotype of colorectal cancer that is contiguous with conventional adenomas, but not serrated polyps. Oncol Lett. 2014;8(5):1937-44. doi:10.3892/ol.2014.2430.
34. Li X, Hu F, Wang Y. CpG island methylator phenotype and prognosis of colorectal cancer in Northeast China. Biomed Res Int. 2014;2014:236361. doi:10.1155/2014/236361.

35. Shiovitz S, Bertagnolli MM, Renfro LA, Nam E, Foster NR, Dzieciatkowski S, et al. CpG island methylator phenotype is associated with response to adjuvant irinotecan-based therapy for stage III colon cancer. Gastroenterology. 2014;147(3):637-45. doi:10.1053/j.gastro.2014.05.009.

36. Wang Y, Long Y, Xu Y, Guan Z, Lian P, Peng J, et al. Prognostic and predictive value of $\mathrm{CpG}$ island methylator phenotype in patients with locally advanced nonmetastatic sporadic colorectal cancer. Gastroenterol Res Pract. 2014;2014:436985. doi:10.1155/2014/436985.

37. Ward RL, Cheong K, Ku SL, Meagher A, O'Connor T, Hawkins NJ. Adverse prognostic effect of methylation in colorectal cancer is reversed by microsatellite instability. J Clin Oncol. 2003;21(20):3729-36. doi:10.1200/jco.2003.03.123.

38. Ju HX, An B, Okamoto Y, Shinjo K, Kanemitsu Y, Komori K, et al. Distinct profiles of epigenetic evolution between colorectal cancers with and without metastasis. Am J Pathol. 2011;178(4):1835-46. doi:10.1016/j.ajpath.2010.12.045.

39. Kakar S, Deng G, Smyrk TC, Cun L, Sahai V, Kim YS. Loss of heterozygosity, aberrant methylation, BRAF mutation and KRAS mutation in colorectal signet ring cell carcinoma. Mod Pathol. 2012;25(7):1040-7. doi:10.1038/modpathol.2012.44.

40. Donada M, Bonin S, Barbazza R, Pettirosso D, Stanta G. Management of stage II colon cancer - the use of molecular biomarkers for adjuvant therapy decision. BMC Gastroenterol. 2013;13:36. doi:10.1186/1471-230x-13-36.

41. Van Rijnsoever M, Elsaleh H, Joseph D, McCaul K, lacopetta B. CpG island methylator phenotype is an independent predictor of survival benefit from 5-fluorouracil in stage III colorectal cancer. Clin Cancer Res. 2003;9(8):2898-903.

42. Ogino S, Meyerhardt JA, Kawasaki T, Clark JW, Ryan DP, Kulke MH, et al. CpG island methylation, response to combination chemotherapy, and patient survival in advanced microsatellite stable colorectal carcinoma. Virchows Arch. 2007;450(5):529-37. doi:10.1007/s00428-007-0398-3.

43. Shen L, Catalano PJ, Benson III AB, O'Dwyer P, Hamilton SR, Issa J-PJ. Association between DNA methylation and shortened survival in patients with advanced colorectal cancer treated with 5-fluorouracil-based chemotherapy. Clin Cancer Res. 2007;13(20):6093-8.

44. Jo P, Jung K, Grade M, Conradi L-C, Wolff HA, Kitz J, et al. CpG island methylator phenotype infers a poor disease-free survival in locally advanced rectal cancer. Surgery. 2012;151(4):564-70.

45. Han SW, Lee HJ, Bae JM, Cho NY, Lee KH, Kim TY, et al. Methylation and microsatellite status and recurrence following adjuvant FOLFOX in colorectal cancer. Int J Cancer. 2013;132(9):2209-16. doi:10.1002/ijc.27888.

46. Herman JG, Graff JR, Myohanen S, Nelkin BD, Baylin SB. Methylation-specific PCR: a novel PCR assay for methylation status of CpG islands. Proc Natl Acad Sci U S A. 1996;93(18):9821-6.

47. Eads CA, Danenberg KD, Kawakami K, Saltz LB, Blake C, Shibata D, et al. MethyLight: a high-throughput assay to measure DNA methylation. Nucleic Acids Res. 2000;28(8):E32.

48. Ehrich M, Nelson MR, Stanssens P, Zabeau M, Liloglou T, Xinarianos G, et al. Quantitative high-throughput analysis of DNA methylation patterns by base-specific cleavage and mass spectrometry. Proc Natl Acad Sci U S A. 2005;102(44):15785-90. doi:10.1073/pnas.0507816102.

49. Xiao Z, Li B, Wang G, Zhu W, Wang Z, Lin J, et al. Validation of methylationsensitive high-resolution melting (MS-HRM) for the detection of stool DNA methylation in colorectal neoplasms. Clin Chim Acta. 2014;431:154-63. doi:10.1016/j.cca.2014.01.044.

50. Ogino S, Kawasaki T, Kirkner GJ, Loda M, Fuchs CS. CpG island methylator phenotype-low (CIMP-low) in colorectal cancer: possible associations with male sex and KRAS mutations. J Mol Diagn. 2006;8(5):582-8. doi:10.2353/jmoldx.2006.060082

51. Shen L, Toyota M, Kondo Y, Lin E, Zhang L, Guo Y, et al. Integrated genetic and epigenetic analysis identifies three different subclasses of colon cancer. Proc Natl Acad Sci U S A. 2007;104(47):18654-9. doi:10.1073/pnas.0704652104.

52. Hinoue T, Weisenberger DJ, Lange CP, Shen H, Byun HM, Van Den Berg D, et al. Genome-scale analysis of aberrant DNA methylation in colorectal cancer. Genome Res. 2012;22(2):271-82. doi:10.1101/gr.117523.110.

53. Lee S, Cho NY, Yoo EJ, Kim JH, Kang GH. CpG island methylator phenotype in colorectal cancers: comparison of the new and classic CpG island methylator phenotype marker panels. Arch Pathol Lab Med. 2008; 132(10):1657-65. doi:10.1043/1543-2165(2008)132[1657:cimpic]2.0.co;2.

54. Ogino S, Kawasaki T, Brahmandam M, Cantor M, Kirkner GJ, Spiegelman D, et al. Precision and performance characteristics of bisulfite conversion and 
real-time PCR (MethyLight) for quantitative DNA methylation analysis. J Mol Diagn. 2006;8(2):209-17. doi:10.2353/jmoldx.2006.050135.

55. Toyooka KO, Toyooka S, Maitra A, Feng Q, Kiviat NC, Smith A, et al.

Establishment and validation of real-time polymerase chain reaction method for CDH1 promoter methylation. Am J Pathol. 2002;161(2):629-34. doi:10.1016/s0002-9440(10)64218-6.

56. de Ruijter TC, de Hoon JP, Slaats J, de Vries B, Janssen MJ, van Wezel T. Formalin-fixed, paraffin-embedded (FFPE) tissue epigenomics using Infinium HumanMethylation450 BeadChip assays. Lab Invest. 2015;95(7):833-42. doi:10.1038/labinvest.2015.53.

57. Phipps Al, Limburg PJ, Baron JA, Burnett-Hartman AN, Weisenberger DJ, Laird PW, et al. Association between molecular subtypes of colorectal cancer and patient survival. Gastroenterology. 2014. doi:10.1053/.j.gastro.2014.09.038.

Submit your next manuscript to BioMed Central and we will help you at every step:

- We accept pre-submission inquiries

- Our selector tool helps you to find the most relevant journal

- We provide round the clock customer support

- Convenient online submission

- Thorough peer review

- Inclusion in PubMed and all major indexing services

- Maximum visibility for your research

Submit your manuscript at www.biomedcentral.com/submit
Biomed Central 